\title{
Survey on Design and Implementation of Indoor Environment Monitoring and control system
}

\author{
Hashi Haris ${ }^{1}$ \\ ${ }^{1}$ Sree Buddha College of Engineering, APJ Abdul Kalam Technological University, \\ Elavumthitta, Kerala 689625
}

\begin{abstract}
:
The ideology of the Internet of things has emerged due to the consolidation of multiple technologies. IoT devices are a part of the larger concept of home automation, also known as domotics. The application of Internet of Things in indoor environment monitoring and control has become a major area as people paying more attention to quality of environment. Intelligent solutions are proposed by studying key technologies of IoT. The system provides a new application for IoT.
\end{abstract}

Keywords: IoT, sensor, automation, web server.

\section{Introduction}

Internet of Things is one of the hot topics of concern in information technology. Many applications such as smart home, intelligent environment monitoring emerged from the concepts of IoT. The system focuses on the abnormality of current management of rooms that leads to disintegration of work force. Indoor environment monitoring and control system would be an acceptable solution to it.

The system uses a number of sensors to get the environmental datas. These datas are passed to Raspberry Pi via $\mathrm{I}^{2} \mathrm{C}$ port. Then they are passed through serial port and GPIO hardware communication technology. The hardware is controlled by Raspberry Pi so as to make adaptive response after analyzing and interacting with the server via socket.

\section{Related Works}

As of 2016, the motivation for the Internet of Things derived from technologies including ubiquitous wireless communication, real-time analytics, machine learning, commodity sensors and embedded systems. In short it means that Internet of Things is enabled by the wireless sensor networks, automation which involves home and building automation, control systems which are the traditional fields of embedded systems.

\subsection{Intelligent Building Developed Model}

The concept of Intelligent Building has become a trending research topic within the engineering community. With the

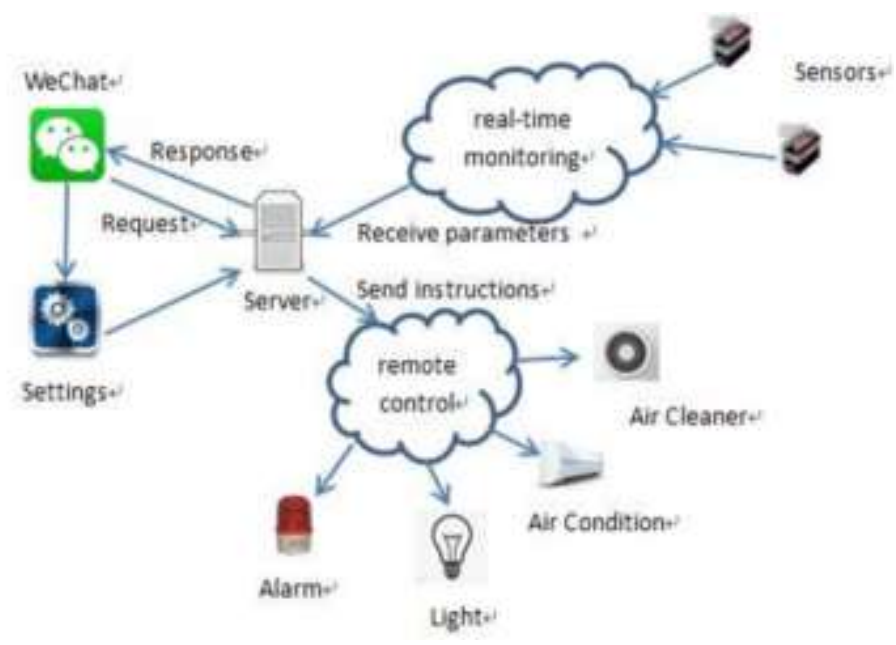

Fig 1. Architecture of the proposed system

development in the standard of living of human beings, there is an increase in the demand for comfortable, convenient, safer and operationally efficient residency. The IB concept can be described as a development model : Inhabitants, Information, Energy and Adaptaion [1]. The term "intelligent" means that the IB s must be able to put up and proceed towards the permanent challenges of environment and alter accordingly to the typical and organizational requirements. The development of "green buildings" was a major action of building industry and building automation. The energy 
utilization is also another goal behind the development of IBs. This can be achieved by a greater usage of HVAC automation systems, enhancing the indoor environmental control and making sure a environment friendly approach towards the entire IB. The future development of IB can be a feasible solution in design and implementation efforts. To organize the Intelligent Buildings better a web based integration model has been developed which is unconstrained of platform, protocol and language by acquiring remote control [2]. For the improvement of the intelligent level of the buildings this integration model is developed referred to as BAS integration. The web based integration model clear the way for the applications of cloud computing in intelligent building. When a greater number of smart devices are being connected to IB networks it has to overcome certain challenges, inoder to provide authentication and key agreement between controller and smart devices a secure SDN framework named $S^{2}$ Net is proposed [3]. This provides security to the IB network with acceptable performance loss. Certain protocols like OpenFlow is designed for the exchange of informations.

\subsection{Intelligent Management System Prototype Design}

A single unified management system is created with the help of technology and processes so as the building can act intelligently making it safer, efficient and comfortable to the users. This can be achieved by designing the system into four layers: physical layer, communication layer, data processing layer, application layer [4]. The physical layer comprises of certain hardware components like the sensors, microcontrollers and actuators. This layer identifies the objects such as temperature and collects information and reacts against the actuator device. The communication layer passes data from physical layer to data processing layer and then from data processing layer to the application layer. The data processing layer stores the data or information from the physical layer. The application layer provides the users with all kinds of applications required by them. Intelligent management system prototype design covers the hardware and software implementations. This system can turn on and off the room lights automatically, detect the fire, automatically operate the regulator of air conditioners, detect and display the room temperature, detect the home with the help of surveillance cameras, provide notification if someone entered the house, detect movement in house, etc.

\subsection{Wireless Sensor Network}

The indoor environment monitoring system detects the temperature, humidity, concentration of formaldehyde and benzene. This system is designed to figure out the quality of indoor environment with the help of multi-sensor data fusion algorithm [5]. ARM11 processor S3C6410 and embedded Linux operating system is used along with the two level fusion algorithm. The benefit is that it provides good accuracy and reliability for evaluation and control of indoor environment. This system can be able to provide long distance data communication. The system has small volume and less power consumption. This system is apt for the environment monitoring.

Smart homes have become a major part in the intelligent building and life. With the advancement of technology along with computers, mobile phones and other communication devices the modern smart homes have been developed. For instance it allows people to control their home equipments even if they are not within the vicinity of their home by just making a hit on the phone gently. The smart home can do various other jobs like detecting the power usage within the home, operating the air conditioner, detecting for any gas leakage, etc. Other than that it also notifies if any intruder is detected to avoid theft and other security risks. The design is done using the ZigBee wireless sensor networks and PLC combination of information transmission. A hybrid network of ZigBee is used inorder to provide flexibility and to blow away the traditional cable complexity. These all are achieved by using mobile phones remote control. This system also uses a double power supply design ideas for stability and reliability.

\subsection{Design and Implementation of smart home based on IoT}

A smart home security monitoring system using the wireless mode has become a major research topic. The existing applications of wireless communication technologies in smart homes are: Infrared, ZigBee, Bluetooth technologies. Infrared technology is short range, half duplex and it uses point to point communication, but it has high error rate so it cannot be used in home networking. Bluetooth is costly and has only limited network capacity so it cannot be 
used in home networking, whereas ZigBee has average transmission distance, greater security and a large networking capacity. A hybrid network design combining ZigBee-based wireless sensor networks and power line carrier network is used in implementation of smart homes [6]. GSM module is used to communicate with the user by notifying the user through sms about any anonymous situation.

\subsection{Design and Implementation of Smart home using Raspberry Pi}

The design of the indoor environment monitoring system is composed of two parts: hardware and software. The major role of hardware (sensors) is to collect the indoor environmental informations which includes the temperature, humidity, carbon dioxide, etc and sent it to the software as parameters. The software is the user interface that receives and analyzes the collected parameters and according to that controls the hardware to how to respond. Inorder to meet the real time requirements hardware is connected to server using a socket connection and software maintains an http connection. The users register the WeChat and they fill the $\mathrm{WiFi}$ account and password thus keeping a socket connection between the Raspberry Pi and server after that they get all services provided by the intelligent indoor environment monitoring and control system [7].

\section{Conclusion}

Smart home and its indoor environment monitoring has become a main research topic, not only that the technologies with the help of which this system can be implemented is another hotspot of research. This survey paper has discussed so far a few number of such technologies and about the concept of a smart home. The future enhancement of the indoor environment monitoring can be emphasized in more areas. By improving the interfaces and integrating more sensors we can make the system more reliable and efficient so that it can be practically a beneficial one.

\section{References}

[1] Cs. Szász, G. Husi, "The Intelligent Building Definition: a CentralEuropean Approach",
Proceedings of the 2014 IEEE/SICE International Symposium on System Integration, 2014,pp.216-221

[2] Yinbo Wu, "An open Web-based integrated system for intelligent building" '(2013)'pp.173-176.

[3] Nian Xue,"S2Net: A Security Framework for Software Defined Intelligent Building Networks"'(2016)'pp.654-661.

[4] Azka Ihsan Nurrahman,Kusprasapta Mutijarsa ,'Intelligent Home Ma nagement System Prototype design and development", Proceedings of ICITSI.2015, pp.1-6, November 2015

[5] Lianjin Guo ' Guosheng Wang ' "Design for Indoor Environment Monitoring System based on Embedded System and Multi-sensor Data Fusion Algorithm"'International Journal of Smart Home' Vol. 10, No. 1, (2016), pp. 31-40

[6] Hu Tao, Zhang Ding and Zhu Shuang-Dong, "A hybrid sensor network in the application design of smart home," Proceedings of IWACI.2010, pp. 437-441, August 2010.

[7] Zhi-xiao Tu, Cheng-chen Hong and Hao Feng,"EMACS: Design and Implementation of Indoor Environmental Monitoring and Control System,"Proceedings of ICIS, 2017 IEEE/ACIS, 2017,pp. 305-309

\section{Author Profile}

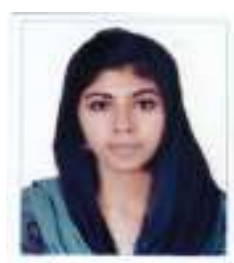

Hashi Haris received the B.tech degree in Computer Science and Engineering from Sree Buddha College of Engineering in 2017 . She is currently pursuing Master's Degree in Computer Science and Engineering in Sree BuddhaCollege of Engineering, Kerala, India. Her research area of interest includes the field of Internet of things, data mining and data analytics in Department of Computer Science and Engineering. 